\title{
Motivações para o prolongamento da amamentação
}

Motivations for breastfeeding prolongation

Motivaciones para la prolongación de la lactancia

Gloria Martínez-Poblete ${ }^{1}$ ib htps://orcid.org/00000-0003-4402-0460

Ximena Ossa ${ }^{2}$ il https://orcid.org/0000-0003-2626-0946

Como citar:

Martínez-Poblete G, Ossa X. Motivaçōes para 0 prolongamento da amamentação. Acta Paul Enferm. 2020;33:eAPE20190112.

DOI

http://dx.doi.org/10.37689/actaape/2020A00112

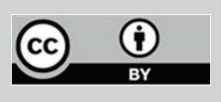

Descritores

Aleitamento materno; Relaç̃̃es mãe-filho; Comportamento alimentar

Keywords Breastfeeding; Child-mother relations; Feeding behavior

Descriptores

Lactancia materna; Relaciones madre-hiijo Conducta alimentaria

Submetido

8 de Maio de 2019

Aceito

11 de Novembro de 2019

\section{Autor correspondente}

Ximena Ossa

E-mail: Ximena.ossa@ufrontera.c

\section{Resumo}

Objetivo: explicar a estrutura motivacional que acompanha a decisão das mães que amamentam por mais de dois anos.

Métodos: estudo qualitativo em mulheres de zonas urbanas e rurais, entre e 20 e 44 anos de idade, nas comunas de Temuco e Padre Las Casas, Chile. A técnica empregada para coleta de dados foi a de entrevista em profundidade. 0 método indutivo de análise, baseado na Teoria Fundamentada em Dados (Grounded Theory), possibilitou um processo comparativo constante até alcançar a saturação teórica dos dados e as três etapas de codificação: aberta, axial e seletiva.

Resultados: oito mulheres concordaram em participar. Foram identificadas quatro dimensões: experiência de vida, autoanálise materna, estímulos da própria mãe e do filho e estímulos sociais e culturais, que estão subjacentes à estrutura motivacional que acompanha a decisão das mães de continuarem amamentando por mais de dois anos. Na infância, as motivações são principalmente intrínsecas (cultura familiar da amamentação). Na idade adulta, há motivações intrínsecas (sentimentos maternos, estímulos internos da mãe) e motivações transcendentes (estímulos da criança), que são reforçadas por estímulos originários do meio social e cultural (ambiente familiar).

Conclusão: para as mulheres, a infância é o período motivacional por excelência para integrar a amamentação como a melhor opção para alimentar seus filhos. Na idade adulta, a motivação transcendente se consolida no primeiro estágio da educação dos filhos e proporciona maior qualidade motivacional ao prolongamento do aleitamento materno. As políticas públicas devem focar suas ações nessas etapas da vida da mulher para melhorar os indicadores de amamentação.

\section{Abstract}

Objective: Our aim was to explain the motivational structure that accompanies the decision by mothers who breastfeed for more than two years.

Methods: Qualitative study in urban and rural women aged 20 to 44 years in the communes of Temuco and Padre Las Casas, Chile. The data collection technique was an in-depth interview. The inductive method of analysis based on Grounded Theory enabled a constant comparative process until the theoretical saturation of the data and the 3 coding stages: open, axial and selective.

Results: Eight women agreed to participate. Four dimensions were identified: life experience, maternal selfanalysis, stimuli from the mother herself and from the child, and social and cultural stimuli, which underlie the motivational structure that accompanies the decision by mothers to continue breastfeeding for longer than two years. In childhood the motivations are mainly intrinsic (family culture of breastfeeding) and in adulthood 
there are intrinsic motivations (maternal feelings, mother's internal stimuli) and transcendent motivations (stimuli from the child), with these being reinforced by stimuli originating from the social and cultural milieu (family surroundings).

Conclusion: For a woman, childhood is the motivational period par excellence to integrate breastfeeding as the best option for feeding her children. In adulthood, the transcendent motivation is consolidated in the first stage of child-rearing, affording greater motivational quality to the prolongation of breastfeeding. Public policies must focus their actions on these stages of a woman's life to improve breastfeeding indicators.

\section{Resumen}

Objetivo: Explicar la estructura motivacional que acompaña la decisión de madres que amamantan durante más de dos años.

Métodos: Estudio cualitativo en mujeres de zonas urbanas y rurales, entre 20 y 44 años de edad, en las comunas de Temuco y Padre Las Casas, Chile. La técnica empleada para la recolección de datos fue la de entrevistas en profundidad. El método inductivo de análisis, basado en la teoría fundamentada en datos (Grounded Theory), posibilitó un proceso comparativo constante hasta alcanzar la saturación teórica de los datos y las tres etapas de codificación: abierta, axial y selectiva.

Resultados: Ocho mujeres aceptaron participar. Se identificaron cuatro dimensiones: experiencia de vida, autoanálisis materno, estímulos de la propia madre y del hijo y estímulos sociales y culturales, que están por detrás de la estructura motivacional que acompaña la decisión de las madres de continuar amamantando durante más de dos años. En la infancia, las motivaciones son principalmente intrínsecas (cultura familiar de la lactancia). En la edad adulta, hay motivaciones intrínsecas (sentimientos maternos, estímulos internos de la madre) y motivaciones trascendentes (estímulos del niño), que se refuerzan con estímulos originarios del medio social y cultural (ambiente familiar).

Conclusión: Para las mujeres, la infancia es el período motivacional por excelencia para integrar la lactancia como la mejor opción para alimentar a sus hijos. En la edad adulta, la motivación trascendente se consolida en el primer nivel de la educación de los hijos y proporciona mayor calidad motivacional para la prolongación de la lactancia materna. Las políticas públicas deben centralizar sus acciones en estas etapas de la vida de la mujer para mejorar los indicadores de lactancia.

\section{Introdução}

O leite materno muda sua composição ao longo do tempo e se adapta às necessidades da criança; e quanto mais prolongada a amamentação, maiores os benefícios para mãe e a criança. ${ }^{(1,2)}$ No entanto, é cada vez menos viável encontrar díades com períodos prolongados de amamentação. ${ }^{(3)}$ Não existe uma definiçãa acordada em relação à amamentação prolongada, mas é considerada acima do período mínimo recomendado pela Organização Mundial da Saúde (OMS), ou seja, dois anos de idade. ${ }^{(4)}$ Atualmente, cerca de $43 \%$ das crianças são amamentadas exclusivamente por seis meses em diferentes partes do mundo. ${ }^{(5)} \mathrm{Na}$ América Latina e no Caribe, a prevalência é de apenas 32\% . ${ }^{(6)}$ No Chile, a prevalência de aleitamento materno exclusivo até o sexto mês é de $53 \%$ e na região de La Araucanía, no centro-sul do país, é superior a 60\%, uma das taxas mais altas do Chile. ${ }^{(7)}$ Esse número é influenciado pela grande população indígena (Mapuche) presente na região: elas frequentemente amamentam até os 2 ou 3 anos de idade. ${ }^{(8)}$ Dados sobre amamentação além dos 2 anos de idade na população em geral são desconhecidos.

No Chile, todos os esforços atuais têm como objetivo melhorar os indicadores de amamentação até o sexto mês, mas náo seu prolongamento ao longo do tempo, apesar do aumento das evidências sobre os benefícios da amamentação prolongada. ${ }^{(1,3,9,10)}$

Existem evidências significativas sobre os motivos de as mães pararem de amamentar. ${ }^{(11,12)}$ Mas poucos estudos revelam as motivaçóes para continuar amamentando além do período culturalmente aceito. As motivaçóes das mães acabam por definir a duração da amamentação. Essa decisão é influenciada por diversos fatores e variadas experiências. De acordo com a teoria da autodeterminação, há motivações que levam as pessoas a agirem de acordo com seus próprios valores e autossatisfação sem que isso leve a uma compensação externa (motivação intrínseca); e motivações provocadas por estímulos externos associados a algum tipo de recompensa material ou reconhecimento social (motivação extrínseca). Ambas as motivaçóes regulam a tomada de decisóes, mas a possibilidade de internalizar e integrar atividades motivadas extrinsecamente com as motivações próprias também promove comportamentos na direção do alcance de uma meta. ${ }^{(13)}$ Finalmente, a motivação transcendente que emerge da área organizacional pode ser extrapolada para forças individuais, que levam as pessoas a atingir objetivos além da autorrealização ou de possíveis recompensas - como o bem-estar de outras pessoas, neste caso, do filho ou filha. ${ }^{(14)}$ A maioria das campanhas ou estratégias de adesão à amamentação se baseia em motivar os profissionais de saúde a pro- 
mover o aleitamento, mas não há uma política clara para empregar as motivaçóes das próprias mulheres, pois há pouca evidência de quais são essas motivaçóes. Esse conhecimento é relevante para que ela seja incorporada no planejamento e na implementação de estratégias para prolongar a amamentação e, com ela, os benefícios para mães e filhos. ${ }^{(3,15)}$

O objetivo deste estudo foi compreender a estrutura motivacional que acompanha a decisão das mães em zonas urbanas e rurais da Região de La Araucanía, Chile, de continuar a amamentação por mais de dois anos.

\section{Métodos}

Um estudo qualitativo com amostragem teórica ${ }^{(16,17)}$ foi realizado durante o segundo semestre de 2016 com mulheres de zonas urbanas e rurais, com idade entre 20 a 44 anos, que compareceram a seus exames de saúde no serviço público ou que pertenciam a uma organização não governamental chamada Trawün Domo (organização social focada na proteção da díade em processo de maternidade e paternidade), localizada nas comunas de Temuco e Padre Las Casas, na regiáo de La Araucanía, Chile. A amostra foi formada até alcançar a saturaçáo dos dados. Era requisito ter amamentado o último filho por mais de dois anos e ter assinado um termo de consentimento livre e esclarecido. $\mathrm{O}$ único critério de exclusão foi incapacidade cognitiva, e não era necessário ler e escrever, pois a técnica de coleta de dados era oral. Esta amostragem é a mais apropriada para explorar novas áreas de conhecimento: privilegia o fenômeno do estudo e, consequentemente, as pessoas que vivem a experiência e podem contribuir melhor para o desenvolvimento do conteúdo. Esse tipo de amostragem maximiza as oportunidades de desenvolver e descobrir variaçóes entre os conceitos e de tornar as categorias mais densas em termos de propriedades e dimensóes. ${ }^{(17)}$ Há poucas mulheres com essas características e a real prevalência de amamentação prolongada é desconhecida.

A técnica de coleta de dados foi a entrevista detalhada, cara a cara. As principais questóes que nortearam a entrevista foram elaboradas com base nos objetivos da pesquisa. ${ }^{(17)}$ As questóes foram testadas em três mulheres, de diferentes níveis culturais e socioeconômicos, para avaliar a compreensão e a objetividade das respostas. As perguntas que levaram a erros de interpretação foram modificadas e reformuladas até serem totalmente compreensíveis. As principais perguntas foram: "Na sua infância e adolescência, você se lembra se sua mãe amamentou você ou algum de seus irmãos?"; "Alguma outra experiência nesse sentido?"; "O que isso significou para você e como isso influenciou sua atual decisão de prolongar a amamentação?"; "Como adulta, que outras experiências você teve?"; "Como elas influenciaram suas decisóes?"; "Durante a gravidez, você recebeu informações sobre amamentação? De quem?"; "Como essas informaçóes influenciaram sua decisão de prolongar a amamentação?"; "Como foi o processo de parto e pós-parto em relação à amamentação?”; "Como essas experiências influenciaram sua decisão?”; "Durante os primeiros dois anos do seu bebê, qual foi sua experiência em relação a aleitamento, assistência à saúde, interação com a família e seu ambiente?"; "De todas essas experiências, o que você acha que mais influenciou sua decisão de amamentar e prolongar a lactação e por quê?"; "O que significou para você continuar amamentando até hoje?”. Essas perguntas foram seguidas por outras, dependendo das respostas, o que permitiu aprofundar o desenvolvimento das categorias. As entrevistas foram realizadas pelo pesquisador principal em locais diferentes, dependendo da preferência dos participantes: sala clínica da unidade de saúde em zona urbana ou rural; nas residências dos participantes ou em sala de reunióes da universidade. Cada entrevista durou entre 45 e 60 minutos. Todos os participantes foram entrevistados na presença do filho/a. As narrativas, em áudio, foram gravadas e transcritas literalmente. O método indutivo de análise foi baseado na Teoria Fundamentada e possibilitou um processo comparativo constante até alcançar a saturação teórica de dados e as três etapas de codificação: aberta, axial e seletiva. ${ }^{(18,19)}$ A partir desse processo, foram construídas diferentes categorias, que foram inter-relacionadas para construir um referencial explicativo com as motivaçóes para o prolongamento da amamentação. A análise qualitativa foi realizada com auxílio do programa Atlas.ti 7. Os resultados foram discutidos com os participantes, confirmando a 
veracidade dos relatos. Este estudo foi aprovado pelo Comitê de Ética Científico da Universidad de La Frontera (N²36/2015).

\section{Resultados}

Das oito mulheres que concordaram em participar, apenas uma possuía menos de oito anos de educação formal, uma trabalhava fora de casa e sete delas tinham um parceiro. Quatro residiam em área urbana, duas pertenciam ao grupo étnico Mapuche e o tempo de amamentaçáo variou entre dois e sete anos. A tabela 1 mostra as características de cada participante.

Foram identificadas quatro dimensôes inter-relacionadas para compor a motivaçáo para prolongar a amamentação: 1) experiências de vida; 2) sentimentos/emoçôes positivas; 3) estímulos da própria mãe e da criança; 4) incentivo social e cultural. Primeiro, foi realizada uma análise dos relatos das mulheres do grupo étnico Mapuche separadamente. Como não foram encontrados elementos diferenciadores originários da cosmovisão em relação às motivaçóes para prolongar a amamentação, foi realizada a análise conjunta. $\mathrm{Na}$ dimensão 'experiências de vida', as ideias foram agrupadas para reconhecer o estágio da vida em que ocorreu a motivação para prolongar a amamentação. As entrevistadas revelaram memórias de terem sido amamentadas por um período prolongado em suas próprias infâncias e de famílias com uma cultura de amamentação prolongada. Alguns relatos representando essa categoria são descritos a seguir:

“...sim, eu tenho uma familia grande. Minha avó deu a luz a nove filhos, sete partos em casa. Da minha mãe, sou a mais velha e somos em seis. Então, eu vi minha máe cuidar de todos os meus irmãos e irmãs. O que veio depois de mim foi amamentado por quatro anos..."

“...sim, a primeira memória que me ocorre é que eu fui amamentada até cerca de cinco (anos), até que meu irmão mais novo começou a mamar e então eu pensei em retomar a mesma cena, mas com meu próprio filho..."

$\mathrm{Na}$ adolescência, a história da cultura familiar foi novamente destacada. No entanto, na vida adulta, surgiram as experiências dos períodos pré-natal, em torno do parto e pós-natal em relação ao prolongamento da amamentação. Também foi destacada a história de ter amamentado um filho anterior por um período prolongado. Os relatos mais comuns nessa categoria foram:

“... não foi que eu determinei antes que iria amamentar, porque há mães que decidiram... quando ela (bebê) nasceu, a primeira coisa que senti foi que ela estava com fome e era instinto. Então, eu disse para a minha mãe que iria amamentar até um ano ... depois pensei ... vamos esperar um pouco mais e no final, decidi, enquanto ela puder, até que ela vá para a pré-escola, até os três ou quatro anos, enquanto ela quiser, eu vou amamentar..."

$\mathrm{Na}$ dimensão 'sentimentos/emoçóes positivas', as mães realizaram autoanálise e descreveram os sentimentos surgidos pela amamentação prolongada e que as levaram a continuar. Emoçôes e sentimentos como felicidade, carinho, orgulho e integridade se destacaram, conforme apresentado nos seguintes relatos:

“...para mim, é algo espetacular. Significa tantas coisas que eu náo consigo nem dar um nome. Pessoalmente, adoro, acho que sou viciada em ocito-

Tabela 1. Características das participantes

\begin{tabular}{|c|c|c|c|c|c|c|c|}
\hline Participantes & Idade & Nível educacional & Residência & Organização & Mapuche & Estado civil & Meses de lactação \\
\hline 1 & 25 & Universidade & Urbana & Trawun Domo & Não & Solteira & 26 \\
\hline 2 & 20 & Ensino médio & Urbana & Unid. Saúde & Não & Casada & 30 \\
\hline $3^{*}$ & 37 & Ensino médio & Urbana & Trawun Domo & Não & Casada & 30 \\
\hline 4 & 36 & Ensino médio & Urbana & Trawun Domo & Não & Casada & 28 \\
\hline 5 & 37 & Ensino fund. I & Rural & Unid. Saúde & Sim & Casada & 34 \\
\hline 6 & 44 & Ensino médio & Rural & Unid. Saúde & Sim & Casada & 84 \\
\hline 7 & 37 & Ensino médio & Rural & Unid. Saúde & Não & Casada & 35 \\
\hline 8 & 26 & Universidade & Rural & Unid. Saúde & Não & Casada & 40 \\
\hline
\end{tabular}


cina (risos), realmente viciada, mas é verdade, nada me deixa tão feliz quanto saber que eu dou o melhor de mim, o melhor que posso dar de mim, eu dou... "

"Eu me sinto orgulhosa... e além disso, eu fui mãe mais velha (risos), então nunca me ocorreu comprar uma lata de fórmula... eles nunca beberam, eu sempre bebia muito leite, então eu disse ia amamentar até ... o máximo que eu pudesse, apesar de terem me dado bronca em casa"

A dimensão 'estímulos da própria mãe e da criança' revela preferências e benefícios que ela percebe por si mesma. A mãe valoriza as necessidades da criança em detrimento das suas, mas também aprecia o conforto sentido na amamentaçáo. Adicionado aos estímulos da criança, está o efeito da amamentação prolongada na saúde e no vínculo da criança. Alguns depoimentos que representam essa dimensão são:

"...mais do que tudo, continuo amamentando porque fui máe solteira e sinto que ela é a coisa mais importante para mim..."

“...o leite materno é muito bom para eles, no sentido de complementar a alimentação, ou seja, vitaminas, minerais, cálcio, hidratação, não é apenas alimento e hidratação, também tem o carinho, sabe?"

“...para mim, é muito mais fácil dar de mamar para dele, especialmente quando saímos, em vez de carregar uma mamadeira, a garrafa térmica com água, então, isso também me ajuda..."

A dimensão 'incentivo social e cultural' do meio ambiente demonstra como a família, a comunidade e os profissionais de saúde apoiam a decisão de prolongar a amamentação. Alguns relatos que descrevem isso:

"...meu marido, ele olha a gente e adora. Ele me apoia em tudo, observa e adora ver Pedro, nosso filho, como ele coloca a cabeça para mamar ...... e para o resto da familia, é algo natural ... não é uma questão...tomar mamadeira seria uma questão"
"Meus vizinhos, por exemplo, não me dizem nada, é algo normal para eles. Minha sogra me disse que antes era comum amamentar porque é um alimento natural; por esse motivo, as crianças de antigamente náo ficavam doentes como agora porque eram amamentadas"

"...o pediatra me disse, dê leite materno, se você tiver leite suficiente, não pare de dar..."

A motivação que acompanha a decisão das mães em zonas urbanas e rurais que amamentam por mais de dois anos pode ter suas origens em suas experiências de vida nos diferentes estágios da vida. $\mathrm{Na}$ infância e adolescência, há uma motivação intrínseca, desencadeando estímulos a partir das lembranças de terem sido amamentadas por um período prolongado e, portanto, de pertencer a famílias onde a amamentação era praticada de maneira natural e espontânea. Este é o ponto de maior relevância em sua decisão. $\mathrm{Na}$ idade adulta, motivaçóes intrínsecas e transcendentes são produzidas nos períodos pré-natal, em torno do parto e pós-natal. Os estímulos sociais e culturais reforçam essas motivaçóes durante todos esses períodos, sendo a mais relevante a decorrente do ambiente familiar. O período pós-natal é quando a maioria dos estímulos é acionado, produzindo a motivação para prolongar a amamentação. Nesse estágio, os estímulos internos (conforto e prioridades da vida) e os sentimentos e emoções (orgulho, nutrição, plenitude, felicidade) despertados pela amamentação fazem parte da motivação intrínseca, diferentemente dos estímulos da criança, que estruturam a motivação transcendente. Ambos os tipos de motivação reforçam e se conectam a outras experiências na fase adulta para tomar a decisão de prolongar a amamentação. Tudo isso constitui a estrutura conceitual motivacional para o prolongamento da amamentação. A figura 1 mostra as categorias, subcategorias, principais códigos e as inter-relaçôes entre eles, que representam o que foi explicado anteriormente. Os números abaixo de cada um refletem as vezes em que apareceram nas entrevistas e as vezes em que são relacionados a outros conceitos que formam as categorias. 


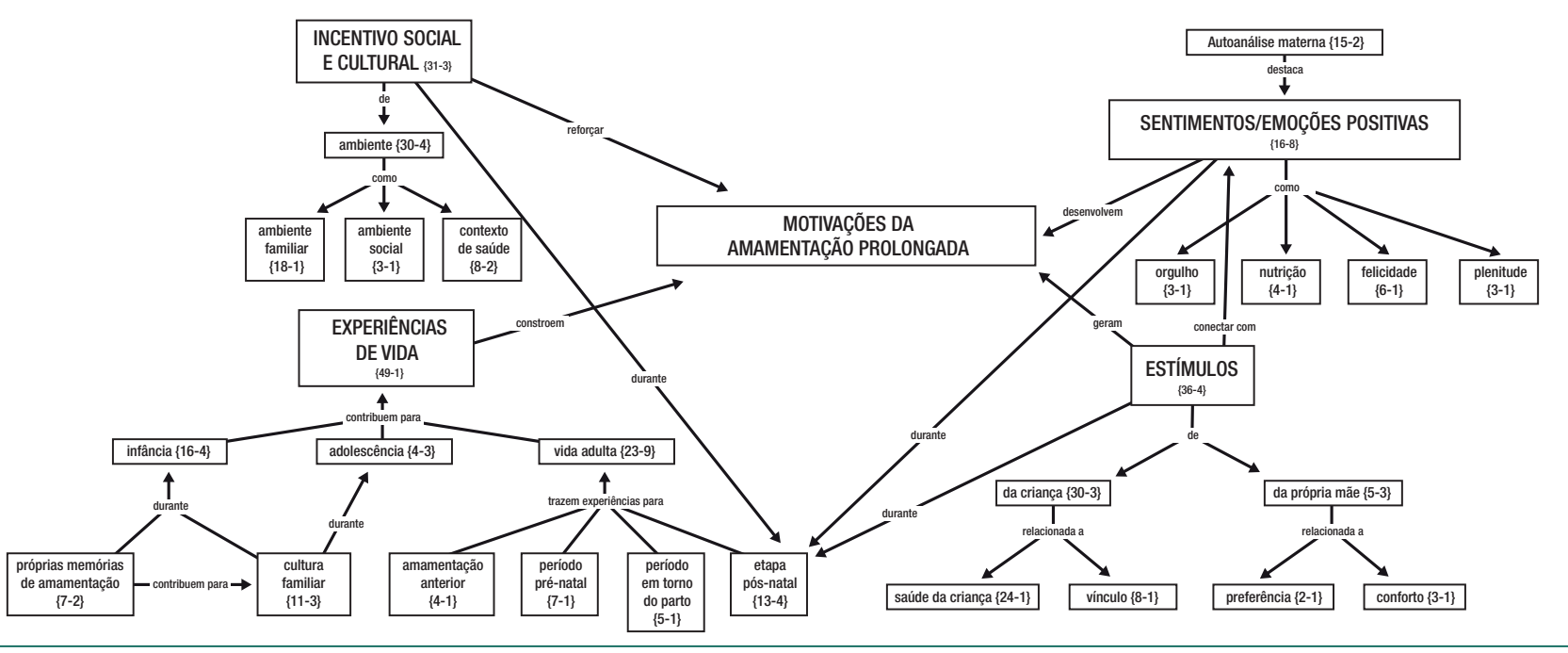

Figura 1. Estrutura motivacional para amamentação prolongada

\section{Discussão}

A motivação como construto hipotético-teórico que alude a um processo complexo causador de um comportamento tem sido amplamente estudada nas áreas de educação e organizaçóes do trabalho, ${ }^{(19-23)}$ mas não na área de tomada de decisão em torno de aspectos da educação infantil como o prolongamento da amamentação.

Entre as mulheres do grupo étnico Mapuche, a amamentação é culturalmente valorizada como uma forma de manter o vínculo e a comunicação com a criança, razão pela qual ocorre de forma natural e espontânea. As práticas típicas desse grupo étnico continuam até hoje, e algumas crianças Mapuches são amamentadas até os seis ou sete anos de idade. (8) Atualmente, esse achado ocorre com menos frequência, particularmente em mulheres que não são da etnia Mapuche, o que dificulta definir a motivação dessas mulheres desde a infância.

Quando a motivação para amamentar por um período prolongado é desenvolvida na idade adulta, particularmente na fase de criação dos filhos, o histórico de uma experiência anterior de amamentação prolongada é relevante. Isso é consistente com Gribble, que demonstrou que 94\% das mulheres com experiência prévia de aleitamento afirmaram que o ato produziu efeito positivo em sua relação atual com a amamentação. ${ }^{\text {(24) }}$
A motivação na idade adulta é reforçada por estímulos da criança (percepção de uma criança mais saudável) e pelo vínculo estabelecido na díade. Alguns resultados bem-sucedidos de amamentação revelam opiniôes maternas semelhantes. ${ }^{(25,26)}$ No entanto, também há resultados com percepçôes maternas ambivalentes, sentimentos agradáveis e outros nem tanto, gerados pela perda de autonomia que acompanha o prolongamento do período. ${ }^{(27)}$ Por outro lado, o conforto para as mães (motivação intrínseca) inclina a escala em favor do prolongamento. Este dado está em consonância com o estudo de Carrascoza et al., destacando que as mães continuam amamentando pelo conforto e facilidade proporcionados pela prática ao saírem em público com seus filhos sem a necessidade de levar utensílios para alimentá-los. ${ }^{(28)}$

Os sentimentos positivos que permitem às mães descreverem a si mesmas como autorrealizadas em seus papéis maternos e de mulher reforçam suas decisões de prolongar a amamentação. Também as tornam imunes aos comentários de seu ambiente sobre o desmame, associados a mitos referentes ao valor nutricional do leite materno. Nenhuma delas reportou sentimentos negativos espontaneamente, ao contrário do relatado por Newman e Williamson, em que a maioria das mulheres disse perceber o estigma social pela amamentação de filhos mais velhos, ${ }^{(29)}$ provavelmente devido a diferenças culturais entre os países.

Os estímulos sociais e culturais fortalecem e aprimoram as motivações intrínsecas e transcendentes na 
idade adulta. $\mathrm{O}$ marido, ou companheiro, e as avós aparecem como figuras importantes no ambiente familiar - apesar de evidências sobre uma influência negativa das avós, principalmente nos primeiros meses de vida, pois levam ao desmame e, em algumas ocasióes, estimulam a introdução da fórmula. ${ }^{(30)}$ Por outro lado, há estímulos dos profissionais de saúde. As mulheres recebem apoio para amamentar durante a gravidez e até os primeiros 6 meses de vida do bebê, quando as indicaçôes são reforçadas. A partir daí, é percebido declínio no reforço e, na realidade, às vezes o desmame é promovido. Isso é percebido como falta de conhecimento dos profissionais de saúde em relação aos benefícios do prolongamento da amamentação. Esses dados também aparecem em outros estudos, em que as mães entrevistadas sugerem maior preparo e informaçóes dos profissionais de saúde com relação à duração do aleitamento materno. ${ }^{(31)}$

A motivação para amamentar e continuar ao longo do tempo foi internalizada e integrada desde o início, de forma intensa e indubitável. Como essa maneira de estruturar a motivação gerou perdas, uma vez que apenas 20\% continuam amamentando até um ano de vida, ${ }^{(6)}$ essa cultura precisa ser recriada na família, nas organizaçóes de saúde e na sociedade, incorporando aspectos cotidianos da amamentaçáo em brinquedos e jogos infantis, no conteúdo da educação formal e da mídia de massa desde o início da infância. ${ }^{(32)}$ Se estas fossem políticas públicas, seria um passo adiante e sem grandes custos para o Estado, o que permitiria a criação de um ambiente cultural coerente de apoio às mães no aleitamento materno e na sua duração. ${ }^{(33,34)}$ As intervençóes do setor de saúde nos estágios pré-natal e pós-parto são particularmente valiosas na ausência da integração da motivação na infância.

Uma limitação deste estudo foi o número de participantes, dada a dificuldade de encontrar máes que amamentam além de dois anos. No entanto, as mulheres entrevistadas, com diferentes características e experiências, contribuíram com riqueza e variabilidade para os relatos e consistência nas semelhanças em relação às suas motivaçóes. Quando a saturação teórica de uma das categorias estava incompleta, os participantes que mencionaram esses aspectos foram entrevistados novamente para aprofundar suas respostas e concluir a análise das dimensóes em que determinadas categorias estavam envolvidas. Isso valida os resultados expressos no referencial conceitual que estrutura a motivação para prolongar a amamentação além dos dois anos no século XXI.

\section{Conclusão}

Esses resultados levam à conclusão de que as motivaçôes intrínsecas e transcendentes são a base para a tomada de decisão no prolongamento do aleitamento. Mulheres que integraram a amamentação prolongada como algo natural na infância não precisaram de incentivos adicionais. No entanto, motivar o prolongamento do período de amamentação deve ser acompanhado de forma mais consistente pelos benefícios associados. O parceiro, ou outro membro familiar significativo para a mulher, também deve ser incluído com mais ênfase como agente ativo e fortalecedor. Essas açôes deveriam ser incluídas nos exames de saúde e nas diretrizes e recomendaçôes. Por um lado, reforçariam as motivaçóes pré-estabelecidas e, por outro, estimulariam motivaçóes intrínsecas e transcendentes em mulheres que tiveram pouca exposição a experiências anteriores. Embora a motivação extrínseca não se aplique a esse aspecto da criação dos filhos, talvez, se a sociedade alcançar uma cultura de amamentação tão relevante, em que mães que prolongam a amamentação tenham reconhecimento social importante, essa motivação também possa ser incluída.

\section{Agradecimentos}

Os autores agradecem ao Programa de Mestrado em Saúde Pública Comunitária e Desenvolvimento Local da Universidad de La Frontera, Temuco, Chile, e à Gloria Martinez-Poblete pelo apoio à pesquisa. Agradecimentos também ao Departamento de Pesquisa da Universidad de La Frontera (DIUFRO) pelo apoio financeiro.

\section{Colaborações}

Ambos os autores contribuíram para: 1. Concepção, desenvolvimento, análise e interpretação dos dados; 
2. Redação do artigo e revisão crítica do conteúdo intelectual relevante; 3. Aprovação final da versão a ser publicada.

\section{Referências}

1. Horta BL, de Sousa BA, de Mola CL. Breastfeeding and neurodevelopmental outcomes. Curr Opin Clin Nutr Metab Care. 2018;21(3):174-8.

2. Leon-Cava N, Lutter C, Ross J, Martin L. Cuantificación de los beneficios de la lactancia materna: reseña de la evidencia [Internet] Washington (DC): Organización Panamericana de la Salud; 2002. 186p. [cited 2019 Jan 6]. Available from: http://iris.paho.org/ xmlui/bitstream/handle/123456789/18539/9275123977_esp. pdf? sequence=1\&isAllowed=y

3. Victora CG, Bahl R, Barros AJ, França GV, Horton S, Krasevec J, et al.; Lancet Breastfeeding Series Group. Breastfeeding in the 21st century: epidemiology, mechanisms, and lifelong effect. Lancet. 2016;387(10017):475-90.

4. World Health Organization and UNICEF. Global strategy for infant and young child feeding [Internet]. Geneve: WHO; 2003. 30p. [cited 2019 Jan 6]. Available from: http://www.who.int/nutrition/publications/gs_ infant_feeding_text_eng.pdf

5. Fondo de las Naciones Unidas para la Infancia. A partir de la primera hora de vida [Internet]. New York, USA: Fondo de las Naciones Unidas para la Infancia; 2016. 103p. [cited 2019 Jan 13]. Available from: https://data. unicef.org/wp-content/uploads/2016/08/From-the-first-hour-of-life.pdf

6. Española de Pediatría A. Comité de Lactancia Materna. Lactancia materna en cifras: tasas de inicio y duración de la lactancia en España y en otros países [Internet]. Madrid: Asociación Española de Pediatría; 2016. 9p. [cited 2019 Jan 13]. Available from http://www.aeped.es/ sites/default/files/documentos/201602-lactancia-materna-cifras.pdf

7. de Salud M. Subsecretaría de redes asistenciales. Gobierno de Chile. Orientaciones Técnicas Metas Sanitarias 2017 [Internet]. Chile: Ministerio de Salud; 2016. 49p. [cited 2019 Jan 20]. Available from http://www.araucanianorte.cl/images/PDF-WORD/OT_METAS_ sanitarias_2017-31-09-2016.pdf

8. Sadler M, Obach A. Pautas de Crianza Mapuche [Internet]. Chile: Ministerio de Planificación, Gobierno de Chile; 2006. 78 p. [cited 2019 Jan 20]. Available from http://www.crececontigo.gob.cl/wp-content/ uploads/2015/11/03-Pautas-de-crianza-mapuche.pdf

9. Binns $C$, Lee M, Low WY. The long-term public health benefits of breastfeeding. Asia Pac J Public Health. 2016;28(1):7-14.

10. Goldman AS, Goldblum RM, Garza C. Immunologic components in human milk during the second year of lactation. Acta Paediatr Scand. 1983;72(3):461-2.

11. Ladomenou F, Kafatos A, Galanakis E. Risk factors related to intention to breastfeed, early weaning and suboptimal duration of breastfeeding. Acta Paediatr. 2007;96(10):1441-4.

12. Huang $Y$, Ouyang $Y Q$, Redding SR. Maternal prepregnancy body mass index, gestational weight gain, and cessation of breastfeeding: a systematic review and meta-analysis. Breastfeeding Med. 2019; 14(6):366-74.

13. Ryan RM, Deci EL. La teoría de la autodeterminación y la facilitación de la motivación intrínseca, el desarrollo social y el bienestar. Am Psychol. 2000;55(1):68-78.
14. García A. Una Nueva Teoría de Motivación: El Modelo Antropológico de Juan Antonio Pérez López. Rev Puertorriquena Psicol. 2004;15:123-63.

15. Stuebe AM, Schwarz EB. The risks and benefits of infant feeding practices for women and their children. J Perinatol. 2010;30(3):155-62.

16. Strauss A, Corbin J. Bases de la investigación cualitativa: técnicas y procedimientos para desarrollar la teoría fundamentada. 1a ed. Colombia: Editorial Universidad de Antoquia; 2002. p. 219-35.

17. Moser A, Korstjens I. Series: Practical guidance to qualitative research. Part 3: Sampling, data collection and analysis. Eur J Gen Pract. 2018;24(1):9-18.

18. Glasser BG, Strauss AL. The discovery of Grounded Theory: Strategies for qualitative research. New York, USA: Routledge; 2017.

19. Broc MA. Motivación y rendimiento académico en alumnos de educación secundaria obligatoria y bachillerato LOGSE. Rev Educ. 2006;340:379-414.

20. Howard J, Gagné M, Morin AJ, Van den BroeckA. Motivation profiles at work: A self-determination theory approach. J Vocat Behav. 2016;(9596):74-89.

21. Kusurkar RA, Croiset G, Ten Cate TJ. Twelve tips to stimulate intrinsic motivation in students through autonomy-supportive classroom teaching derived from self-determination theory. Med Teach. 2011;33(12):978-82.

22. Wang WT, Hou YP. Motivations of employees' knowledge sharing behaviors: A self-determination perspective. Inform Organ-UK. 2015;25(1):1-26.

23. Schiefele $U$, Löweke S. The nature, development, and effects of elementary students' reading motivation profiles. read Res $Q$. 2018;53(4):405-21.

24. Gribble KD. Long-term breastfeeding; changing attitudes and overcoming challenges. Breastfeed Rev. 2008;16(1):5-15.

25. Edwards R. An Exploration of maternal satisfaction with breastfeeding as a clinically relevant measure of breastfeeding success. J Hum Lact. 2018;34(1):93-6.

26. Scharfe E. Maternal attachment representations and initiation and duration of breastfeeding. J Hum Lact. 2012;28(2):218-25.

27. Schmied V, Lupton D. Blurring the boundaries: breastfeeding and maternal subjectivity. Sociol Health IIIn. 2001;23(2):234-50.

28. Carrascoza KC, Costa AL Junior, Ambrosano GM, Alves de Moraes $A B$. Prolongamento da amamentação após o primeiro ano de vida: argumentos das mães. Psicol, Teor Pesqui. 2005;21(3):271-7.

29. Newman KL, Williamson IR. Why aren't you stopping now?!' Exploring accounts of white women breastfeeding beyond six months in the East of England. Appetite. 2018;129:228-35.

30. Susin LR, Giugliani ER, Kummer SC. Influence of grandmothers on breastfeeding practices. Rev Saude Publica. 2005;39(2):141-7.

31. Dowling S, Brown A. An exploration of the experiences of mothers who breastfeed long-term: what are the issues and why does it matter? Breastfeed Med. 2013;8(1):45-52.

32. Galvão DM, da Silva IA. Portuguese school children breastfeeding experiences. Rev Esc Enferm USP. 2011;45(5):1055-62.

33. Dornan L, Sinclair M, Kernohan WG, Stockdale J, Khuwuthyakorn V, Suppasan P. Thai cultural influences on breastfeeding behaviour. Evid Based Midwifery. 2015;13(3):84-91.

34. Dettwyler KA. When to wean: biological versus cultural perspectives. Clin Obstet Gynecol. 2004;47(3):712-23. 